\title{
Risk factors of severe hemoptysis in patients with fungus ball
}

\author{
Tae Hoon Kim ${ }^{1}$, Hyun Jung Koo ${ }^{2}$, Chae-Man Lim $^{3}$, Sang-Bum Hong ${ }^{3}$, Jin Won Huh ${ }^{3}$, Kyung Wook Jo ${ }^{3}$, \\ Tae Sun Shim ${ }^{3}$, Woo Sung Kim³ , Younsuck Koh ${ }^{3}$
}

${ }^{1}$ Department of Internal Medicine, Gyeongsang National University School of Medicine and Gyeongsang National University Changwon Hospital, Changwon, Republic of Korea; ${ }^{2}$ Department of Radiology, ${ }^{3}$ Department of Pulmonary and Critical Care Medicine, Asan Medical Center, University of Ulsan College of Medicine, Seoul, Republic of Korea

Contributions: (I) Conception and design: TH Kim, Y Koh; (II) Administrative support: Y Koh; (III) Provision of study material or patients: All authors; (IV) Collection and assembly of data: TH Kim, HJ Koo, Y Koh; (V) Data analysis and interpretation: TH Kim, HJ Koo, Y Koh; (VI) Manuscript writing: All authors; (VII) Final approval of manuscript: All authors.

Correspondence to: Younsuck Koh. Department of Pulmonary and Critical Care Medicine, Asan Medical Center, University of Ulsan College of Medicine, 88, Olympic-ro 43-gil, Songpa-gu, Seoul 05505, Republic of Korea. Email: yskoh@amc.seoul.kr.

Background: Hemoptysis is one of the most important complications associated with fungus ball. However, the association of the primary size of the fungus ball and its change with the incidence of hemoptysis was not fully established. This study aims to determine the clinical risk factors of severe hemoptysis and natural course of fungus ball.

Methods: A retrospective study on patients with fungus ball was performed. Patients with severe hemoptysis-related events were compared to those without events, and the predictors for severe hemoptysis were investigated by Cox regression analysis. Three subgroups (decreased, stable, and increased) were classified according to the long-term size change, and the severe hemoptysis-free survival duration was analyzed by the Kaplan-Meier survival curve.

Results: Among 173 patients, 50 (28.9\%) experienced severe hemoptysis-related events. Risk-adjusted analysis revealed that age [hazard ratio (HR), 0.964; 95\% confidence interval (CI), 0.939-0.989], bloodtinged sputum [HR, 2.717; 95\% CI, 1.513-4.879], and thick wall cavitary fungus balls [HR, 2.332; 95\% CI, 1.290-4.215] were significant predictors of severe hemoptysis-related events in patients with fungus ball. However, the baseline size of the fungus ball and its change rate were not independent risk factors for severe hemoptysis-related events. Besides, the decreased group did not have a favorable outcome in severe hemoptysis related to fungus ball.

Conclusions: In a tertiary hospital, the incidence of severe hemoptysis-related events in patients with fungus ball was not low and reached $28.9 \%$. Young patients, those with thick wall cavitary fungus ball, and those with complaints of blood-tinged sputum should be considered for early surgical treatment to prevent severe hemoptysis.

Keywords: Fungus ball; hemoptysis; aspergilloma; outcome; surgery

Submitted Apr 03, 2019. Accepted for publication Sep 18, 2019.

doi: $10.21037 /$ jtd.2019.09.52

View this article at: http://dx.doi.org/10.21037/jtd.2019.09.52

\section{Introduction}

Fungus ball is an incidental radiologic finding that can be called as aspergilloma. The clinical course of fungus ball has a wide spectrum, from spontaneous resolution to lifethreatening hemoptysis (1). Hemoptysis is one of the most important complications associated with fungus ball, with an incidence of up to $93-95 \%(2,3)$. Also, the mortality rate for aspergilloma-associated hemoptysis ranged from $5 \%$ to $14 \%$ (4-6). However, the relationship between the characteristics of fungus ball and hemoptysis is not well established. A previous study showed that major hemoptysis 
was not related to the size of a fungus ball and the nature of the preexisting lung disease (4). In contrast, some studies reported the association of primary size of fungus ball and its change with the incidence of hemoptysis (7-9).

Hemoptysis due to fungus ball has been managed frequently by bronchial arterial embolization (BAE), which can provide immediate response; however, a nonbronchial systemic artery can also be a bleeding focus in fungus ball (10), and BAE may be ineffective in some cases. Therefore, some studies recommended surgical treatment as the treatment of choice (11-13). However, fungus ball is frequently associated with various predisposing conditions, including pulmonary tuberculosis, sarcoidosis, bronchiectasis, pneumothorax, fibrosis, abscess, cysts, and lung cancer $(11,14,15)$. As these underlying diseases can disturb the natural course of a fungus ball, prognostication and identification of the optimal timing for surgical resection remain as challenges. Moreover, for surgical resection to be feasible, a proper lung function is necessary.

The present study aimed to identify the risk factors of fungus ball-related severe hemoptysis under clinical supervision at a tertiary referral hospital and to investigate the natural course of fungus ball. A clear understanding of these issues may help in clinical decisions on the management of patients with fungus ball, including the timing of surgical resection.

\section{Methods}

\section{Study population and design}

This retrospective study was approved by the Institutional Review Board (2016-0357), which waived the need for informed consent. We utilized radiologic reports in the clinical research database from January 2000 to October 2015 to find out patients with fungus ball. The 3,792 cases of chest computed tomography (CT) reports were discovered, and the clinical and radiological data for 2,026 patients were obtained. Eligible patients were those who had clinical symptoms and radiologic evidence of slowly progressive fungus ball, including cavities and intra-cavitary mass with the surrounding crescent of air (16), those who were over 18 years and had over 2 times chest CT scans with 3 years interval. Exclusion criteria were a limited measurement of fungus ball due to motion artifacts, poorly expanded lung, invasive aspergillosis, patients diagnosed to have other diseases during the follow-up period, and patients with the previous history of massive hemoptysis.
After the application of the inclusion criteria, 173 patients were enrolled and analyzed (Figure 1). One pulmonologist and one thoracic radiologist measured the size of each fungus ball. The size of a fungus ball was calculated from serial CT scans, using length, width, and height. As assuming that the fungus ball is an ellipsoid, the volume formula was $\mathrm{V}=$ $4 / 3 \pi$ (length $/ 2 \times$ width $/ 2 \times$ height $/ 2$ ). Patients were divided into three groups (9), according to changes in fungus ball volume, as determined between the initial-to-last ratio of fungus ball volume. The "decreased group" comprised cases in which the size of the fungus ball shrunk to more than half of the original size, whereas the "increased group" included cases in which the size of the fungus ball doubled from the original size. The remaining cases were classified in the "stable group." To investigate morphologic feature of fungus ball, various radiologic variables, including the number of fungus ball, calcification, thick wall cavitary lesion, pleural thickening, perilesional ground-glass opacity, and the number of destroyed lobes, were evaluated. Cavitary wall thickness was estimated by visual assessment. The thickwalled cavity was defined greater than $4 \mathrm{~mm}$, based on data from cavitary lung diseases (17).

\section{Severe hemoptysis definitions}

Severe hemoptysis was defined as more than $100 \mathrm{~mL}$ of coughed out blood, as estimated in the medical records $(18,19)$. Severe hemoptysis-related events included clinically significant hemoptysis events. Thus, the severe hemoptysisrelated events were comprehensively defined as the occurrence of severe hemoptysis, or cases that needed BAE or surgical treatment due to uncontrolled hemoptysis from fungus ball.

\section{Statistical analysis}

Characteristics of the study subgroups were compared using an unpaired $t$-test, Mann-Whitney $U$-test, ANOVA, or Kruskal-Wallis test, as appropriate, for continuous variables, and Fisher's exact test for categorical variables. Severe hemoptysis-free survival duration was defined as the time from initial detection of fungus ball to the first severe hemoptysis-related event. The differences in severe hemoptysis-free survival were assessed by KaplanMeier survival curves and log-rank test. A univariate Cox's proportional hazards regression model, followed by multivariate analysis, was used to determine the risk factors for severe hemoptysis-related events. Variables 


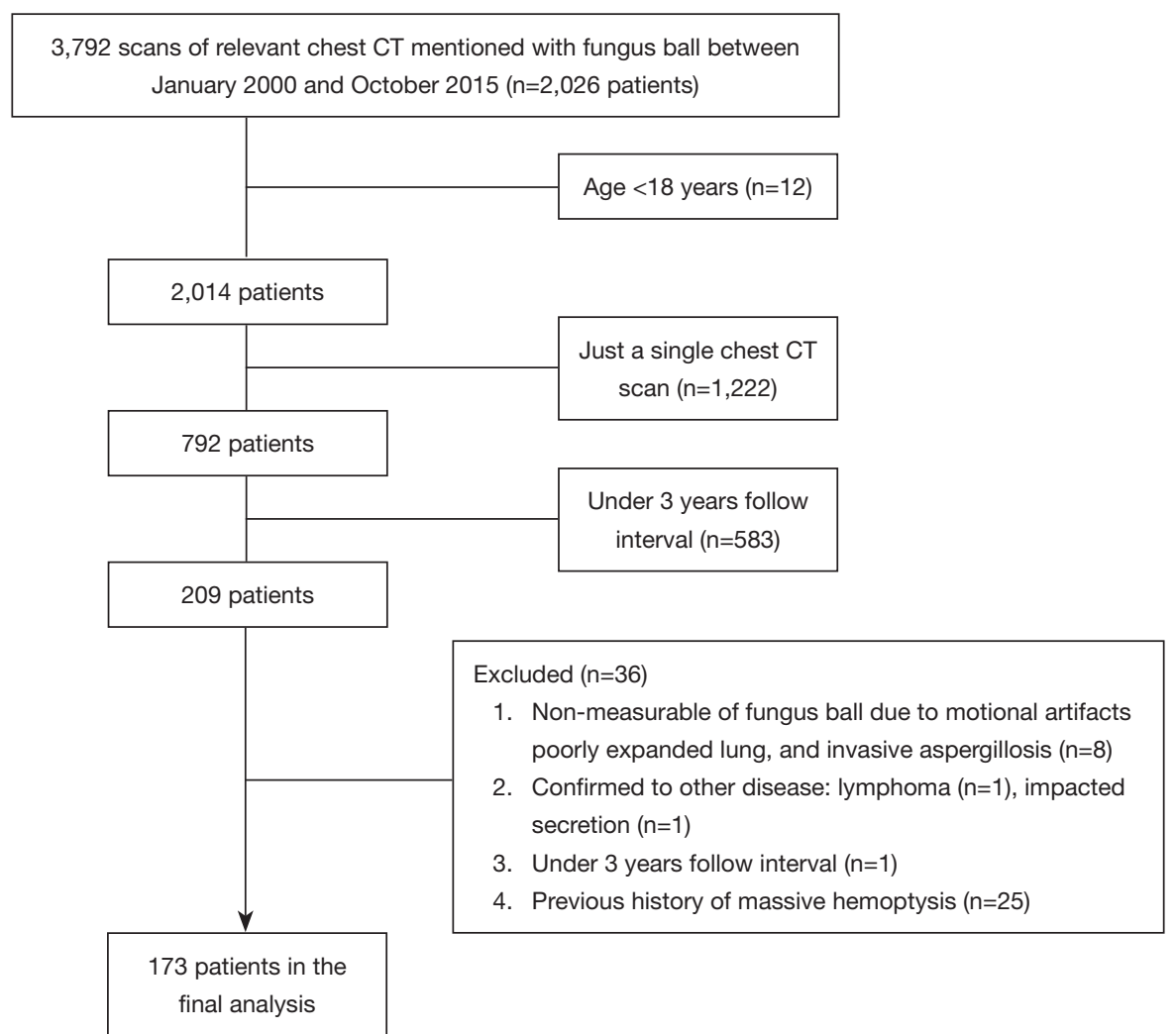

Figure 1 Flow diagram for patient enrollment.

with significance on univariate analysis and previously known risk factors [i.e., age, sex, body mass index (BMI), ever-smoker, past-history of tuberculosis, initial size of fungus ball, multiple fungus ball, thick cavitary lesion, and perilesional ground-glass opacity] (19-21) were evaluated by multivariate analysis with stepwise backward elimination method to determine the independent predictors of severe hemoptysis-related events. Statistical analyses were performed using SPSS 24.0 (IBM Corporation, Armonk, NY, USA) or R version 3.2.3 (R Foundation for Statistical Computing, Vienna, Austria). Data were expressed as mean \pm standard deviation (SD) for the variance of a normal distribution or as the median and interquartile range (IQR) for those with non-normal distribution. For all tests, a value of $\mathrm{P}<0.05$ was considered significant.

\section{Results}

\section{Baseline characteristics of subjects}

Baseline characteristics of the study patients are shown in Table 1. Among 173 patients, 100 (57.8\%) were men and 85 (49.1\%) had a median smoking habit of 30.0 pack-years. Mean age of the patients was 58.1 (range, 23-83) years. Mean predicted values of $\mathrm{FVC}, \mathrm{FEV}_{1}$, and diffusion capacity were $79.3 \%$, $78.5 \%$, and $73.6 \%$, respectively. Some patients had respiratory symptoms, including blood-tinged sputum (30.1\%), cough (39.9\%), purulent sputum (37.6\%), and dyspnea (24.3\%). The most common respiratory comorbidities were bronchiectasis (67.1\%), and sequelae of tuberculosis (46.8\%).

The radiologic features of fungus ball are described in Table 1. The size of fungus balls was median 1.8 (IQR, $0.8-5.9$; mean, $5.3 \pm 11.3) \mathrm{cm}^{3}$. Multiple fungus balls were observed in $30(17.3 \%)$ patients. A thick wall cavitary lesion, pleural thickening, and perilesional ground-glass opacity were observed in $62(35.8 \%), 69(39.9 \%)$, and $11(6.4 \%)$ patients, respectively. Tuberculosis sequelae remained in a median of 2.0 lobes. Chest CT was followed for a median of 5.1 years, and the patient's hemoptysis was monitored for a median of 5.7 years from the initial CT scan.

\section{Severe hemoptysis associated with fungus ball}

Among the study populations, patient with severe hemoptysis- 
Table 1 Baseline characteristics of subjects

\begin{tabular}{|c|c|c|c|}
\hline Baseline characteristics & $\begin{array}{l}\text { Patients without severe hemoptysis } \\
\text { related events }(n=123)\end{array}$ & $\begin{array}{l}\text { Patients with severe hemoptysis } \\
\text { related events }(n=50)\end{array}$ & Total patients $(n=173)$ \\
\hline Age (years) & $60.3 \pm 9.8$ & $52.9 \pm 11.2$ & $58.1 \pm 10.7$ \\
\hline BMI $\left(\mathrm{kg} / \mathrm{m}^{2}\right)$ & $22.6 \pm 3.3$ & $21.7 \pm 3.8$ & $22.4 \pm 3.4$ \\
\hline \multicolumn{4}{|l|}{ Smoking history } \\
\hline Ex-smoker & $38(32.8)$ & $21(42.9)$ & $59(35.8)$ \\
\hline Current-smoker & $19(16.4)$ & $7(14.3)$ & $26(15.8)$ \\
\hline Smoking duration (pack-years) & $30.0(15.0-40.0)$ & $19.8(7.8-33.0)$ & $30.0(10.2-40.0)$ \\
\hline \multicolumn{4}{|l|}{ Past respiratory illness } \\
\hline COPD & $13(10.6)$ & $5(10.0)$ & $18(10.4)$ \\
\hline Interstitial lung disease & $13(10.6)$ & $2(4.0)$ & $15(8.7)$ \\
\hline \multicolumn{4}{|l|}{ Respiratory symptoms } \\
\hline Blood-tinged sputum & $22(17.9)$ & $30(60.0)$ & $52(30.1)$ \\
\hline Cough & $43(35.0)$ & $26(52.0)$ & 69 (39.9) \\
\hline Purulent sputum & $41(33.3)$ & $24(48.0)$ & $65(37.6)$ \\
\hline Dyspnea & $32(26.0)$ & $10(20.0)$ & $42(24.3)$ \\
\hline \multicolumn{4}{|l|}{ Pulmonary functions } \\
\hline FVC, L (\%predicted) & $2.9 \pm 0.8(80.3 \pm 16.5)$ & $2.9 \pm 0.9(77.1 \pm 21.7)$ & $2.9 \pm 0.8(79.3 \pm 18.3)$ \\
\hline Calcification in fungus ball & $43(35.0)$ & $11(22.0)$ & $54(31.2)$ \\
\hline Multiple fungus balls & $15(12.2)$ & $15(30.0)$ & $30(17.3)$ \\
\hline Thick cavitary lesion & $34(27.6)$ & $28(56.0)$ & $62(35.8)$ \\
\hline Pleural thickening & $43(35.0)$ & $26(52.0)$ & 69 (39.9) \\
\hline Perilesional GGO & $4(3.3)$ & $7(14.0)$ & $11(6.4)$ \\
\hline Destroyed lobes ( 1 to 6$)^{a}$ & $2.0(1.0-4.0)$ & $3.0(1.0-5.0)$ & $2.0(1.0-4.0)$ \\
\hline CT follow-up duration ${ }^{a}$ & $4.9(3.8-6.6)$ & $6.0(4.4-9.9)$ & $5.1(3.9-7.3)$ \\
\hline Size change rate $\left(\mathrm{cm}^{3} / \text { year }\right)^{a}$ & $0.1(-0.0-0.6)$ & $0.1(-0.3-0.6)$ & $0.1(-0.1-0.6)$ \\
\hline Decreased group & $15(12.2)$ & $10(20.0)$ & $25(14.5)$ \\
\hline Stable group & $69(56.1)$ & $26(52.0)$ & $95(54.9)$ \\
\hline Increased group & 39 (31.7) & $14(28.0)$ & $53(30.6)$ \\
\hline
\end{tabular}

Subgroups according to volume change is defined as follows: decreased group, final volume $\leq 50 \%$ of initial volume; increased group, final volume $\geq 200 \%$ of initial volume; stable group, final volume $\geq 50 \%$ of initial volume and $\leq 200 \%$ of initial volume. Data are presented as numbers $(\%)$ or mean \pm standard deviation unless otherwise indicated. ${ }^{\text {a }}$, median (interquartile range). BMI, body mass index; COPD, chronic obstructive pulmonary disease; FVC, forced vital capacity; $\mathrm{FEV}_{1}$, forced expiratory volume in 1 second; $\mathrm{DL}_{\mathrm{co}}$, diffusing capacity for carbon monoxide; GGO, ground-glass opacity; CT, computed tomography. 
Table 2 According to size change of fungus ball, difference of severe hemoptysis events

\begin{tabular}{|c|c|c|c|c|}
\hline Hemoptysis related events & Decreased group $(n=25)$ & Stable group $(n=95)$ & Increased group $(n=53)$ & $\mathrm{P}$ \\
\hline Multiple episode of severe hemoptysis & $4 / 10(40.0)$ & 9/19 (47.4) & $7 / 12(58.3)$ & 0.683 \\
\hline Severe hemoptysis (events/year) ${ }^{a}$ & $0.2(0.1-0.3)$ & $0.3(0.2-0.4)$ & $0.4(0.2-0.6)$ & 0.069 \\
\hline BAE & $5(20.0)$ & $11(11.6)$ & $8(15.1)$ & 0.530 \\
\hline Multiple episode of BAE & $3 / 5(60.0)$ & $6 / 11(54.5)$ & $3 / 8(37.5)$ & 0.673 \\
\hline Surgical treatment & $2(8.0)$ & $11(11.6)$ & $7(13.2)$ & 0.798 \\
\hline
\end{tabular}

Subgroups according to volume change is defined as follows: decreased group, final volume $\leq 50 \%$ of initial volume; increased group, final volume $\geq 200 \%$ of the initial volume; stable group, final volume $\geq 50 \%$ of the initial volume and $\leq 200 \%$ of initial volume. Data are presented as numbers $(\%)$ or mean \pm standard deviation unless otherwise indicated. ${ }^{a}$, median (interquartile range). BAE, bronchial arterial embolization.

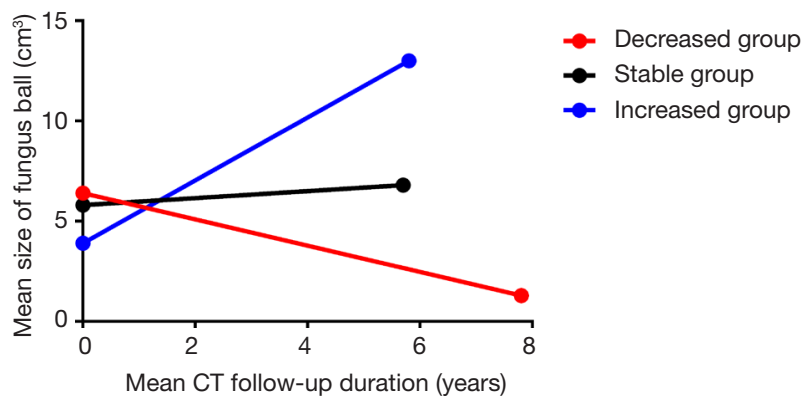

Figure 2 Clinical course of fungus ball. Subgroups according to volume change is defined as follows: decreased group (blue lines), final volume $\leq 50 \%$ of initial size; increased group (red lines), final volume $\geq 200 \%$ of initial size; stable group (black lines), final volume $\geq 50 \%$ of initial volume and $\leq 200 \%$ of initial size.

related events was 50 (28.9\%) in Table 2. Severe hemoptysis was observed in $41(23.7 \%)$ patients, who had a mean 0.3 events per year. Twenty patients of them suffered through multiple severe hemoptysis episodes. Besides, 24 (13.9\%) patients underwent $\mathrm{BAE}$ and $20(11.6 \%)$ patients underwent surgical treatment to control severe hemoptysis associated with fungus ball. During the follow-up period, 14 patients died, but hemoptysis-related mortality was only 1 patient.

\section{Clinical course of fungus ball}

The clinical outcomes according to size change were presented in Table 2 and Figure 2. During a chest CT follow-up, the size of fungus ball was calculated to increase a median of 0.1 (IQR, -0.1 to 0.6 ) $\mathrm{cm}^{3} /$ year. Of 173 patients, $25(14.5 \%)$ patients were in the decreased group, 53 (30.6\%) patients were in the increased group, and others 95 (54.9\%) patients were in the stable group.

The patients with severe hemoptysis-related events in the decreased, stable, and the increased group were as follows; 10 (40.0\%), 26 (27.4\%), and 14 (26.4\%), respectively. There was no difference in the number of patients with severe hemoptysis-related events $(\mathrm{P}=0.413)$, including severe hemoptysis $(\mathrm{P}=0.109)$, $\mathrm{BAE}(\mathrm{P}=0.530)$, and surgical treatment $(\mathrm{P}=0.798)$, among three groups. Kaplan-Meier survival analysis also showed no difference in severe hemoptysisrelated event-free survival duration [Log-rank (Mantelcox), $\mathrm{P}=0.267]$. The estimated mean severe hemoptysisfree survival duration of the decreased, stable, and increased group as follows; 9.7, 12.7, and 10.1 years, respectively.

\section{Severe hemoptysis and its predictive factors}

The clinical features of patients with severe hemoptysisrelated events were shown in Table 1 . These patients were significantly younger $(\mathrm{P}<0.001)$ and had more frequent pasthistory of tuberculosis $(\mathrm{P}=0.002)$ and blood-tinged sputum $(\mathrm{P}<0.001)$. There was no difference in the occurrence of severe hemoptysis-related events in patients with underlying diseases, including hypertension $(\mathrm{P}=0.370)$, heart disease 
Table 3 Prediction of severe hemoptysis in patients with fungus ball

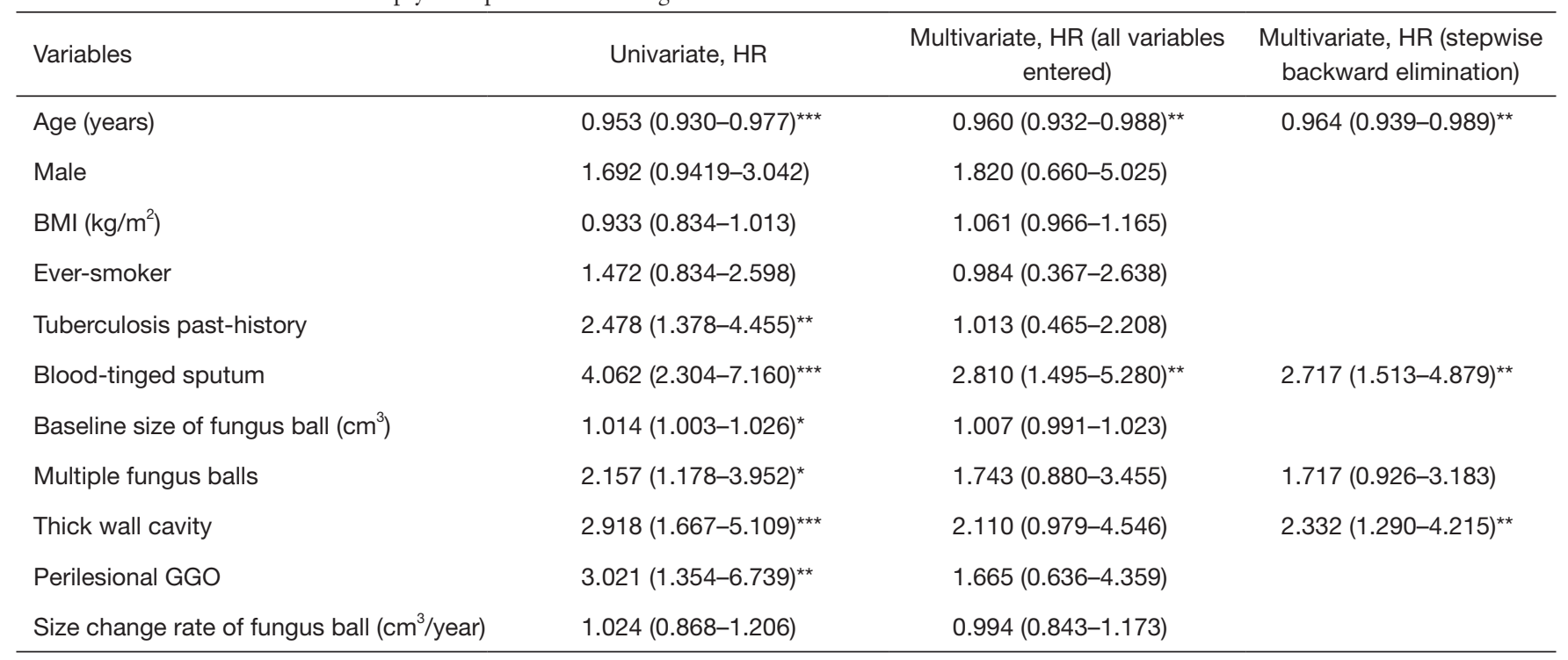

*, $\mathrm{P}<0.05$; ${ }^{* \star}, \mathrm{P}<0.01$; ${ }^{* \star}, \mathrm{P}<0.001$. HR, hazard ratio; BMI, body mass index; GGO, ground-glass opacity.

$(\mathrm{P}=1.000)$, chronic liver disease $(\mathrm{P}=0.180)$, or diabetes $(\mathrm{P}=1.000)$.

Radiologically, larger sized fungus ball $(\mathrm{P}<0.001)$, multiple fungus balls $(\mathrm{P}=0.010)$, thick wall cavitary lesion $(\mathrm{P}=0.001)$ and perilesional ground-glass opacity $(\mathrm{P}=0.022)$, were observed in the patients with severe hemoptysisrelated events, compared to those without events.

To see the predictable factors of severe hemoptysisrelated events in patients with fungus ball, cox regression analysis was performed in Table 3. The univariate analysis showed that young age, past-history of pulmonary tuberculosis, blood-tinged sputum, larger and multiple fungus ball, thick wall cavitary lesion, and perilesional ground-glass opacity were associated with severe hemoptysis-related events. While multivariate analysis showed that those events were independently associated with young age (HR, 0.964; $\mathrm{P}=0.005)$, blood-tinged sputum (HR, 2.717; $\mathrm{P}=0.001)$, and thick wall cavitary fungus ball lesion $(\mathrm{HR}, 2.332 ; \mathrm{P}=0.005)$. Notably, the initial size of fungus ball $(\mathrm{P}=0.400)$ and its change rate $(\mathrm{P}=0.944)$ were not a predictor of severe hemoptysis-related events in riskadjusted analysis.

Among 173 patients, 35 (20.2\%) patients were treated with antifungal agents for a median of 126 days. Twentyfour patients had applied the antifungal agents before severe hemoptysis-related events. There was no difference between the patients to those who were not treated with antifungal agents and those without treatment, by propensity matching analysis $(\mathrm{P}=0.600)$.

\section{Discussion}

We investigated the risk factors of severe hemoptysis in patients with fungus ball and the natural course by longterm observation. Among the study population, 28.9\% of patients developed severe hemoptysis-related events. At risk-adjusted analysis, size of fungus ball and its change rate could not affect the incidence of severe hemoptysis-related events. But, younger age, blood-tinged sputum, thick wall cavitary lesion were revealed as the independent predictors of severe hemoptysis-related events in patients with fungus ball. The size of the fungus ball was increased as a median of $0.1 \mathrm{~cm}^{3} /$ year $(18.4 \pm 39.6 \% /$ year). There was no difference in the severe hemoptysis-related events among groups according to the size change of the fungus ball.

Reports on the association between size of fungus ball and prevalence of hemoptysis are contradicting $(9,22)$. In the present study, patients with severe hemoptysis-related events had larger fungus ball. However, the initial size of the fungus ball was not an independent predictor for severe hemoptysis in the risk-adjusted analysis. The size change rate of the fungus ball also did not relate to the development of severe hemoptysis-related events.

Generally, the prognosis of patients with decreased size of fungus ball may be considered better (7-9). However, the decreased group did not have favorable outcomes in severe 
hemoptysis-related events, compared with the stable or increased group. This finding is consistent with a previous study (9). Besides, in the present study, there was no difference in severe hemoptysis-related events free survival duration among the three groups. In long-term observation, these results suggested that a decrease in the size of a fungus ball did not guarantee an improvement of clinical outcome. The reason for the severe hemoptysis events in patients with reduced fungus ball size is unclear. Active movement and rubbing against the cavity wall of an immature and fragile fungus ball might have led to reduction in its size, along with conversion to an invasive form in some portions of the fungus ball. This assumption was supported by a research on pathology, which showed that a fungus ball is fragile and may be broken easily into fragments; also, the cavity and connecting bronchi were observed to be filled by massive inflammatory exudates and necrotic tissues (20).

Recent studies reported good outcomes of surgical treatment of fungus ball and suggested as the treatment of choice $(11-13,15,23)$. However, the difficulty is the timing of surgical resection (1). In the present study, the risk-adjusted analysis showed that young age, blood-tinged sputum, and thick wall cavitary fungus ball lesion were independent risk factors for severe hemoptysis-related events. Meanwhile, a surgical team also suggested that young age was a predictor of favorable surgical outcome (24). Some papers radiologically classified fungus ball as a simple or complex lesion and explained that the complex fungus ball had a thickwall cavity, surrounding parenchymal disease, and greater pleural thickening $(2,25)$. They showed the resection of the complex lesion had a satisfactory long-term outcome (2). Taken together, if a young patient with a complex fungus ball complains of blood-tinged sputum, this patient may be an appropriate candidate for surgical management.

Old papers have shown that antifungal agents have the effect of attenuating hemoptysis (26,27). Also, there were studies shown that antifungal agents are effective therapeutic tools in patients with chronic pulmonary aspergillosis as well as fungus ball $(8,28)$. They explained that antifungal agents could decrease or stabilize fungus burden. In contrast, other studies showed that systemic antifungal agents were ineffective, because of inadequate drug penetration into the fungus ball and that long therapeutic session may result in poor compliance of the patients $(14,29)$. In the present study, the patients who applied the antifungal agents before severe hemoptysis were too few and had not the constant duration of treatment. Thus, we could not confirm the preventive effect of antifungal agents on severe hemoptysis in patients with fungus ball. Most of the patients were treated with itraconazole. The role of voriconazole, which has recently emerged as the primary option of chronic pulmonary aspergillosis (30), was not evaluated in our study. Besides, we could not confirm the effects of anticoagulant drugs on the development of hemoptysis in fungal patients. Indirectly, in patients with hypertension, heart disease, or diabetes who were more likely to take anticoagulant drugs, there was no difference in the occurrence of severe hemoptysis.

Some limitations of this study include the following. First, this study had a retrospective design and was performed at a single tertiary referral center. Therefore, some selection bias, including more symptomatic patients with fungus ball, could not be avoided. Because of the exclusion of some patients without multiple CT follow-up, less severe and asymptomatic patients might be not included in this study. In addition, patients with previous episodes of massive hemoptysis were also excluded. Therefore, we could not understand the clinical outcomes of these patients. Next, patients with fungus ball were diagnosed by radiologic findings, but not mycology. Thus, there were many diseases, such as actinomycosis, nocardiosis, intracavitary hematoma, and adenocarcinoma, with imaging findings that mimic aspergilloma (31). Although we excluded patients with these mimicking diseases in the follow-up period, this might not be complete. Lastly, most fungus balls were incidentally discovered, so the real-time point of the initial formation of these balls cannot be deciphered. This may have led to time bias in the measurement of severe hemoptysis-related events free survival duration. Change in the size of the fungus was calculated based on the values by CT scan at the beginning and end, so the internal changes in follow-up duration were not reflected the size change rate. The shape of the fungus balls is not constant and there may be a difference between the actual size and the calculated size. Hence, a well-designed prospective study is required to confirm the relationship between the size change rate of fungus balls and severe hemoptysis.

\section{Conclusions}

In a tertiary hospital, the incidence of severe hemoptysisrelated events in patients with fungus ball was not low and reached $28.9 \%$. The size and its change of fungus ball were not an independent risk factor for severe hemoptysis. However, young age, symptom with blood-tinged sputum, and thick wall cavitary lesion were the most significant predictors of severe hemoptysis-related events in patients 
with fungus ball; patients with these predictors might need early surgical treatment.

\section{Acknowledgments}

None.

\section{Footnote}

Conflicts of Interest: The authors have no conflicts of interest to declare.

Ethical Statement: The authors are accountable for all aspects of the work in ensuring that questions related to the accuracy or integrity of any part of the work are appropriately investigated and resolved. This study was reviewed and approved by the Institutional Review Board (2016-0357) of Asan Medical Center.

\section{References}

1. Moodley L, Pillay J, Dheda K. Aspergilloma and the surgeon. J Thorac Dis 2014;6:202-9.

2. Akbari JG, Varma PK, Neema PK, et al. Clinical profile and surgical outcome for pulmonary aspergilloma: a single center experience. Ann Thorac Surg 2005;80:1067-72.

3. Khan MA, Dar AM, Kawoosa NU, et al. Clinical profile and surgical outcome for pulmonary aspergilloma: nine year retrospective observational study in a tertiary care hospital. Int J Surg 2011;9:267-71.

4. Jewkes J, Kay PH, Paneth M, et al. Pulmonary aspergilloma: analysis of prognosis in relation to haemoptysis and survey of treatment. Thorax 1983;38:572-8.

5. Faulkner SL, Vernon R, Brown PP, et al. Hemoptysis and pulmonary aspergilloma: operative versus nonoperative treatment. Ann Thorac Surg 1978;25:389-92.

6. Karas A, Hankins JR, Attar S, et al. Pulmonary aspergillosis: an analysis of 41 patients. Ann Thorac Surg 1976;22:1-7.

7. Godet C, Laurent F, Bergeron A, et al. CT Imaging Assessment of Response to Treatment in Chronic Pulmonary Aspergillosis. Chest 2016;150:139-47.

8. Agarwal R, Vishwanath G, Aggarwal AN, et al. Itraconazole in chronic cavitary pulmonary aspergillosis: a randomised controlled trial and systematic review of literature. Mycoses 2013;56:559-70.

9. Lee JK, Lee YJ, Park SS, et al. Clinical course and prognostic factors of pulmonary aspergilloma. Respirology 2014;19:1066-72.

10. Lee JH, Kwon SY, Yoon HI, et al. Haemoptysis due to chronic tuberculosis vs. bronchiectasis: comparison of long-term outcome of arterial embolisation. Int J Tuberc Lung Dis 2007;11:781-7.

11. Brik A, Salem AM, Kamal AR, et al. Surgical outcome of pulmonary aspergilloma. Eur J Cardiothorac Surg 2008;34:882-5.

12. Aydoğdu K, Incekara F, Sahin MF, et al. Surgical management of pulmonary aspergilloma: clinical experience with 77 cases. Turk J Med Sci 2015;45:431-7.

13. El Hammoumi MM, Slaoui O, El Oueriachi F, et al. Lung resection in pulmonary aspergilloma: experience of a Moroccan center. BMC Surg 2015;15:114.

14. Kawamura S, Maesaki S, Tomono K, et al. Clinical evaluation of 61 patients with pulmonary aspergilloma. Intern Med 2000;39:209-12.

15. Muniappan A, Tapias LF, Butala P, et al. Surgical therapy of pulmonary aspergillomas: a 30-year North American experience. Ann Thorac Surg 2014;97:432-8.

16. Roberts CM, Citron KM, Strickland B. Intrathoracic aspergilloma: role of CT in diagnosis and treatment. Radiology 1987;165:123-8.

17. Ryu JH, Swensen SJ. Cystic and cavitary lung diseases: focal and diffuse. Mayo Clin Proc 2003;78:744-52.

18. Fidan A, Ozdoğan S, Oruc O, et al. Hemoptysis: a retrospective analysis of 108 cases. Respir Med 2002;96:677-80.

19. Lee BR, Yu JY, Ban HJ, et al. Analysis of patients with hemoptysis in a tertiary referral hospital. Tuberc Respir Dis (Seoul) 2012;73:107-14.

20. Ando T, Tochigi N, Gocho K, et al. Pathophysiological Implication of Computed Tomography Images of Chronic Pulmonary Aspergillosis. Jpn J Infect Dis 2016;69:118-26.

21. Sapienza LG, Gomes MJ, Maliska C, et al. Hemoptysis due to fungus ball after tuberculosis: A series of 21 cases treated with hemostatic radiotherapy. BMC Infect Dis 2015;15:546.

22. Lee SH, Lee BJ, Jung DY, et al. Clinical manifestations and treatment outcomes of pulmonary aspergilloma. Korean J Intern Med 2004;19:38-42.

23. Lejay A, Falcoz PE, Santelmo N, et al. Surgery for aspergilloma: time trend towards improved results? Interact Cardiovasc Thorac Surg 2011;13:392-5.

24. Lee JG, Lee CY, Park IK, et al. Pulmonary aspergilloma: analysis of prognosis in relation to symptoms and treatment. J Thorac Cardiovasc Surg 2009;138:820-5. 
25. Battaglini JW, Murray GF, Keagy BA, et al. Surgical management of symptomatic pulmonary aspergilloma. Ann Thorac Surg 1985;39:512-6.

26. Kamei K, Kohno N, Tabeta H, et al. The treatment of pulmonary aspergilloma with itraconazole. Kansenshogaku Zasshi 1991;65:808-12.

27. Impens N, De Greve J, De Beule K, et al. Oral treatment with itraconazole of aspergilloma in cavitary lung cancer. Eur Respir J 1990;3:837-9.

28. Camuset J, Nunes H, Dombret MC, et al. Treatment of chronic pulmonary aspergillosis by voriconazole in nonimmunocompromised patients. Chest

Cite this article as: Kim TH, Koo HJ, Lim CM, Hong SB, Huh JW, Jo KW, Shim TS, Kim WS, Koh Y. Risk factors of severe hemoptysis in patients with fungus ball. J Thorac Dis 2019;11(10):4249-4257. doi: 10.21037/jtd.2019.09.52
2007;131:1435-41.

29. Kravitz JN, Berry MW, Schabel SI, et al. A modern series of percutaneous intracavitary instillation of amphotericin $\mathrm{B}$ for the treatment of severe hemoptysis from pulmonary aspergilloma. Chest 2013;143:1414-21.

30. Cucchetto G, Cazzadori A, Conti M, et al. Treatment of chronic pulmonary aspergillosis with voriconazole: review of a case series. Infection 2015;43:277-86.

31. Gazzoni FF, Severo LC, Marchiori E, et al. Pulmonary diseases with imaging findings mimicking aspergilloma. Lung 2014;192:347-57. 